\title{
Evaluation of planned dosimetry when beam energies are substituted for a fraction of the treatment course
}

\author{
Samantha E Hawke, Angela Torrance, Lindsay Tremethick \\ Genesiscare, Radiation Oncology Victoria, Murray Valley Private Hospital, Wodonga, Victoria, Australia.
}

Received September 11, 2013; Revised October 31, 2013; Accepted November 10, 2013; Published Online November 22, 2013

\section{Technical Report}

\begin{abstract}
Purpose: The purpose of this technical study was to evaluate how the effect of changing beam energies for one to multiple fractions of a patient's plan affected the overall dose delivered to the planning target volume (PTV) and surrounding organs at risk (OAR's). Method: In this study, twenty-eight patient plans from treatment sites including the oesophagus, prostate, lung, spine, rectum, bladder, chest, scapula, and breast were evaluated in the Philips Pinnacle treatment planning system (TPS), of these 14 were originally planned with $15 \mathrm{MV}$ and 14 with $10 \mathrm{MV}$. Each of these plans were substituted with a single to multiple fractions with $10 \mathrm{MV}$ and $15 \mathrm{MV}$ respectively while keeping the original monitor units the same. Results: It was determined that when the number of fractions of the substituted beam energy remained at one fifth or less of the overall fractions a change of dose of less than $2 \%$ to the PTV could be maintained. The OAR's dose, when the plan had $20 \%$ of its fractions substituted with a different energy, were found to change by on average up to $3.5 \%$ and $2.3 \%$ for original plan energies of $15 \mathrm{MV}$ and $10 \mathrm{MV}$ respectively. The dose change calculated in the TPS was then verified using ion chamber measurements for bladder and oesophagus treatment plans. Conclusion: Results appear to indicate that the site of treatment was not an important factor when changing energy but the overall number of fractions versus the number of fractions substituted with an alternative energy was fundamental. These results may be clinically useful when a radiotherapy department have machines with different photon energies. In the event of a break down, when a patient needs to be urgently treated, it may be possible to treat them on another machine with a different energy, without an immediate recalculation in the TPS. This decision would depend upon the percentage of fractions of their overall treatment needing to be treated before the machine was repaired.
\end{abstract}

Keywords: Radiotherapy; Fraction; Treatment planning system; Beam energy; Substitution

\section{Introduction}

Murray Valley Radiation Oncology Centre has two linear accelerators with different photon energies; a Varian 21iX with $6 \mathrm{MV}$ and $10 \mathrm{MV}$ photon beams and a Varian $21 \mathrm{eX}$ with $6 \mathrm{MV}$ and $15 \mathrm{MV}$ photon energies. ${ }^{1}$ Problems arise when patients planned with $10 \mathrm{MV}$ or $15 \mathrm{MV}$ cannot be treated due to the machine with that energy capability breaking down. As this department is in a rural setting the patients cannot be treated in another department due to the distance of travel involved.

Corresponding author: Samantha E Hawke; Genesiscare, Radiation Oncology Victoria, Murray Valley Private Hospital, Nordsvan Dr., Wodonga, Victoria 3690, Australia.

Email: smayers@radoncvic.com.au

Cite this article as:

Hawke S, Torrance A, Tremethick L. Evaluation of planned dosimetry when beam energies are substituted for a fraction of the treatment course. Int J Cancer Ther Oncol 2013; 1(2):01014.

DOI: $10.14319 /$ ijcto.0102.4
Previous studies have compared the same treatment plan being carried out on two separate energies for the entire duration of the treatment. In one study the difference between $6 \mathrm{MV}$ and $18 \mathrm{MV}$ for treatment of lung cancer was evaluated and it was observed that there was no clinically significant difference. ${ }^{2}$ However these plans had been recalculated and optimised in the treatment planning system. It was found in another study that low energy beams provided better conformity to the target than high energy beams due to reduced lateral scatter. ${ }^{3}$

In a more recent study by Molazeda et al. ${ }^{4}$ oesophageal and pelvis plans were compared using both $6 \mathrm{MV}$ and $15 \mathrm{MV}$ where all parameters entered in the treatment planning system (TPS) such as beam angle and weighting were kept the same. It was established that there was no significant difference in uniform dose coverage to the PTV in both the oesophageal and rectum plans when the energy was changed in the TPS. However it should be noted that in the study by Molazeda et al. the plan was recomputed to find the optimal 
monitor unit (MU) from each angle. This differs from what is being conducted in this technical note where all parameters, including the MU's are kept the same, and the plan recomputed to find the difference to the PTV and OAR's.

The purpose of this technical study was to investigate whether a patient could have treatment between one and several fractions of their whole treatment with substituted beam energy and to quantify the difference in dose to the planning target volume (PTV) and organs at risk (OAR).

\section{Methods and Materials}

\section{Computation using TPS}

The TPS used in this study was Pinnacle (Philips Healthcare, Andover, MA) version 9.2.5 A variety of treatment plans generated using three dimensional conformal radiation therapy (3DCRT) were selected from the TPS with treatment sites including the oesophagus, prostate, lung, spine, rectum, bladder, chest, scapula and breast. Of the 28 plans selected half were planned with $15 \mathrm{MV}$ and half with $10 \mathrm{MV}$. The number of fractions ranged from palliative treatments with 5 fractions to radical treatments with 34 fractions. In each case the energy of a single fraction was changed to 15MV if the plan was originally $10 \mathrm{MV}$ and $10 \mathrm{MV}$ if the original plan was $15 \mathrm{MV}$. This was then repeated with 2, 3, 4, 5, 10 and all fractions being changed either to $15 \mathrm{MV}$ or $10 \mathrm{MV}$. The modified treatment plan retained the same number of monitor units as the original plan. The calculation algorithm used in the TPS was collapsed cone convolution with a $0.28 \mathrm{~cm}$ grid size. The percentage changes to the mean dose of the PTV and OAR's were evaluated. The average PTV volume for the plans investigated is shown in Table 1.

TABLE 1: Average PTV volume for plans investigated.

\begin{tabular}{lc}
\hline \hline PTV & Average Volume $\left(\mathrm{cm}^{3}\right)$ \\
\hline Oesophagus & 310.88 \\
Prostate & 185.67 \\
Lung & 928.06 \\
Spine & 1183.85 \\
Rectum & 798.36 \\
Bladder & 741.83 \\
Chest & 606.42 \\
Scapula & 218.62 \\
Breast & 1866.32 \\
\hline \hline
\end{tabular}

\section{Ion chamber measurements}

Two bladder treatment plans were transferred onto a cylindrical phantom. The isocenter of the plans was moved to allow the ion chamber to be located within the PTV. The plan was modified so that trial 1 contained 5 fractions with $15 \mathrm{MV}$ and trial 2 with 4 fractions of $15 \mathrm{MV}$ and 1 fraction of
$10 \mathrm{MV}$. This second trial allowed for $20 \%$ of the treatment plan to be delivered with $10 \mathrm{MV}$. The dose to the isocentres was recorded from the TPS for both trials. Both trials of the oesophagus plans were delivered to the cylindrical phantom with a Wellhofer cc13 chamber (Wellhofer Dosimetrie, Schwrazenbruck Germany) ${ }^{6}$ (with a collecting volume of $0.13 \mathrm{~cm}^{3}$ and previously known as an IC15) inserted at the centre. The ion chamber measurements were then compared with the TPS results.

Similarly two oesophagus treatment plans were transferred to a cylindrical phantom dataset where two plan trials were created. Trial 1 contained 5 fractions of $10 \mathrm{MV}$ and trial 2 contained 4 fractions of $10 \mathrm{MV}$ and 1 fraction of $15 \mathrm{MV}$. Ion chamber measurements from the oesophagus trials were compared to the results from the TPS.
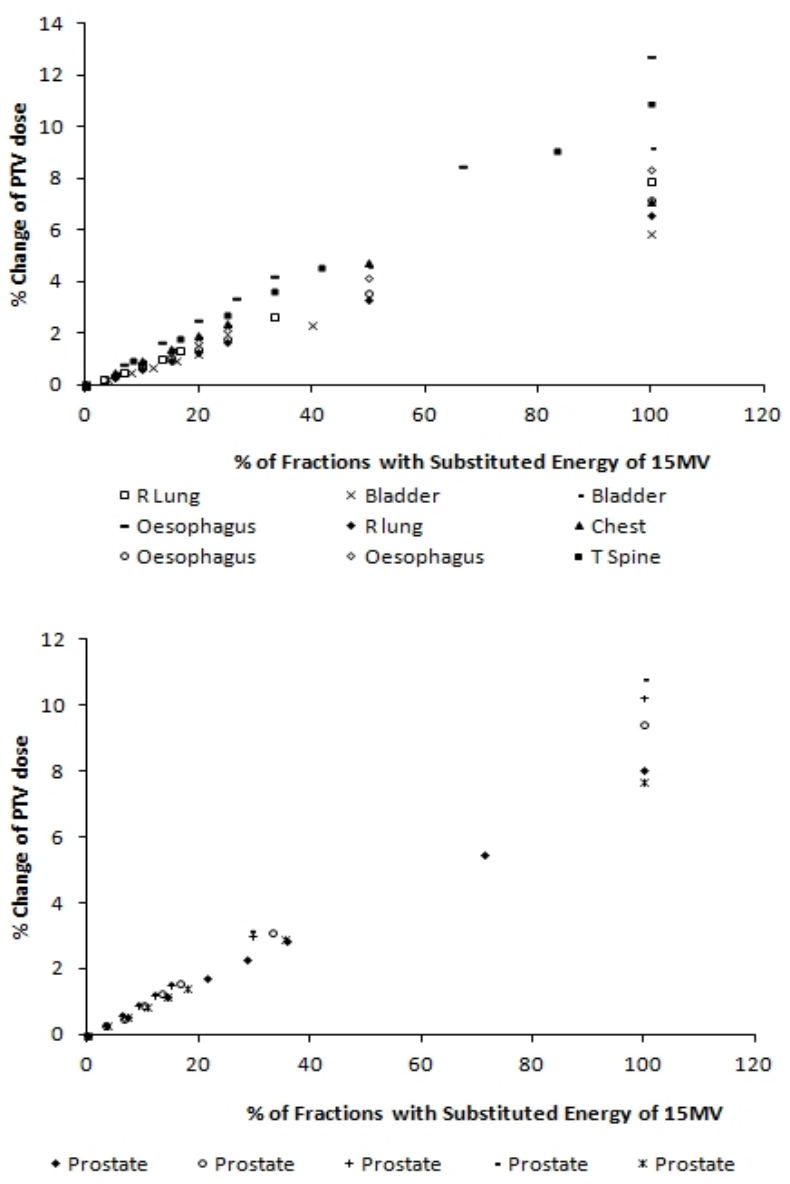

FIG. 1: A plot showing the change in the PTV dose when a percentage of the treatment was substituted with $15 \mathrm{MV}$ instead of the original planned 10MV for (a) three oesophagus plans, a spine plan, two bladder plans, a chest plan and two lung plans and (b) five prostate plans. 


\section{Results}

\section{Computation using TPS}

Each of the patient plans investigated were plotted using the percentage change in dose to the PTV as a function of the percentage of fractions with the substituted energy. These findings are shown in Figure 1 for a $10 \mathrm{MV}$ plan with $15 \mathrm{MV}$ substituted in for various numbers of fractions and Figure 2 where a $15 \mathrm{MV}$ treatment plan had various numbers of fractions substituted with 10MV. Each of the different symbols represents an individual patient plan. The percentage change in the PTV dose was calculated using equation 1 where plan $\mathrm{A}$ is the dose to the PTV in the original plan and plan B is the dose to the PTV in the plan with the substituted fraction/s.

$$
\% \text { changeof PTV dose }=\frac{D_{\text {plan } B}-D_{\text {plan } A}}{D_{\text {plan } A}} \times 100
$$
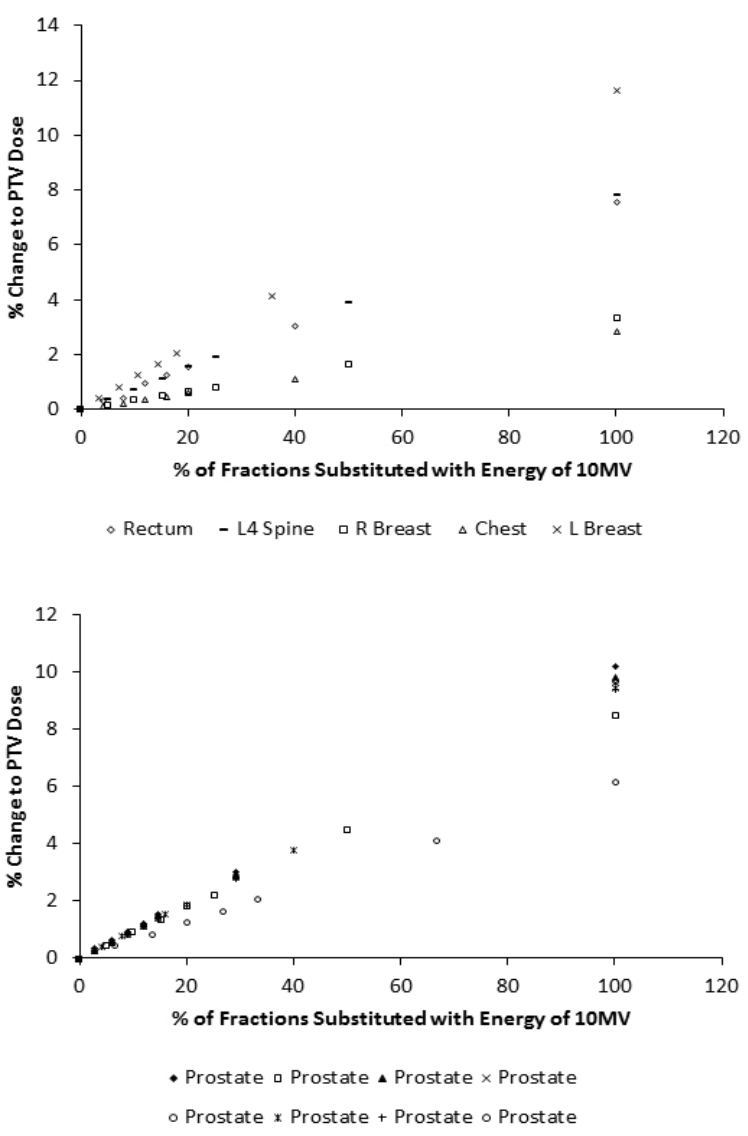

FIG. 2: A plot showing the change in the PTV dose when a percentage of the treatment was substituted with $10 \mathrm{MV}$ instead of the original planned 15MV for (a) a rectum plan, a spine plan, a chest plan and breast plan and (b) eight prostate plans.
The percentage change in the dose to the OARs were tabulated for each treatment plan when a fifth of the fractions had been substituted with $15 \mathrm{MV}$ for a $10 \mathrm{MV}$ plan, Table 1, and $10 \mathrm{MV}$ for a $15 \mathrm{MV}$ plan, Table 2.

TABLE 2: Percentage change for a fifth of the fractions of a 10MV plan substituted with $15 \mathrm{MV}$ for the organs at risk (OARs)

\begin{tabular}{lcc}
\hline \hline Treatment Site & OAR & $\begin{array}{c}\text { Average of changes to } \\
\text { Mean dose \% }\end{array}$ \\
\hline R Lung & Spinal Cord & 0.3 \\
Bladder & Heart & 1.4 \\
& Left femur & 3.9 \\
Oesophagus & Right Femur & 1.9 \\
& Spinal Cord & 1.5 \\
& Heart & 2.9 \\
Chest & Left Lung & 0.5 \\
& Right Lung & 2.5 \\
\multirow{4}{*}{ T- Spine } & Spinal Cord & 1.4 \\
& Spinal Cord & 2.9 \\
& Left Lung & 0.7 \\
& Right Lung & 3.1 \\
Prostate & Bladder & 3.2 \\
& Rectum & 1.8 \\
\hline \hline
\end{tabular}

TABLE 3: Percentage change for a fifth of the fractions of a $15 \mathrm{MV}$ plan substituted with $10 \mathrm{MV}$ for the organs at risk (OARs).

\begin{tabular}{lcc}
\hline \hline Treatment Site & OAR & $\begin{array}{c}\text { Average of changes to } \\
\text { Mean dose \% }\end{array}$ \\
\hline \multirow{4}{*}{ Rectum } & Bladder & 1.5 \\
& Left Femur & 2.3 \\
& Right Femur & 2.1 \\
L4 Spine & Small bowel & 2.1 \\
& Spinal Cord & 0.6 \\
& Left Kidney & 2.3 \\
Breast & Right Kidney & 1.3 \\
& Spinal Cord & 0.4 \\
& Right Lung & 2.4 \\
Chest & Left Lung & 1.4 \\
& Right Lung & 1.2 \\
& Left Lung & 1.5 \\
Prostate & Spinal cord & 0.7 \\
& Bladder & 2.2 \\
\hline \hline
\end{tabular}

\section{Ion chamber measurements}

The TPS results for the bladder and oesophagus treatment plans are compared with ion chamber measurements, shown in Table 4. Trial 1 for the bladder treatment plans refers to 5 fractions of $15 \mathrm{MV}$ and trial 2 refers to 4 fractions of $15 \mathrm{MV}$ and 1 fraction of 10MV. Similarly for the Oesophagus trial 1 refers to 5 fractions of $10 \mathrm{MV}$ and trial 2 refers to 4 fractions of $10 \mathrm{MV}$ and 1 fraction of $15 \mathrm{MV}$. 
TABLE 4: TPS calculation compared to ion chamber measurements for bladder and oesophagus treatment sites

\begin{tabular}{lcl}
\hline \hline Treatment Site & $\Delta^{\mathrm{a}}$ & $\Delta^{\mathrm{b}}$ \\
\hline Bladder & 1.2 & 0.6 \\
Oesophagus & 1.1 & 0.9 \\
\hline \hline
\end{tabular}

Abbreviations: $\Delta^{\mathrm{a}}=$ Average $\%$ difference between ion chamber measurement of Trial 1 and 2; $\Delta^{\mathrm{b}}=$ Average \% difference between TPS and ion chamber measurement

\section{Discussion and Conclusion}

It can be seen from Figure 1 that when the $15 \mathrm{MV}$ beam is substituted in for a $10 \mathrm{MV}$ plan the change to the PTV appears to follow a linear relationship with the number of fractions substituted. A similar phenomenon was also seen in Figure 2. More importantly the majority of the plans when substituted with $15 \mathrm{MV}$ for $20 \%$ of the fractions have a corresponding change in dose to the PTV of $<2 \%$. This is consistent with a study by Pokharel $\mathrm{S}^{7}$ where $6 \mathrm{MV}$ prostate plans where substituted with mixed energy plans of $6 \mathrm{MV}$ and $16 \mathrm{MV}$ and the total dose to the PTV was found to vary by less than $1 \%$ from the original plan.

The International Commission on Radiation Units and Measurements (ICRU) recommend that the PTV dose remains within $+7 \%$ and $-5 \%$ of the prescription dose; therefore when considering if a plan could have one or multiple fractions with a substituted energy the dose to the PTV and the prescription dose needs to be considered. ${ }^{8}$

From Table 2, it is apparent that the average change to the OAR's for a substitution of a $15 \mathrm{MV}$ beam for $20 \%$ of a $10 \mathrm{MV}$ plan varies between $0.3 \%$ to $3.9 \%$. For a $15 \mathrm{MV}$ plan substituted with $10 \mathrm{MV}$ for $20 \%$ of the fractions shows that on average the OAR's have a change of $<2.3 \%$. These findings are detailed in Table 3.

It was determined that up to $20 \%$ of the fractions for a $10 \mathrm{MV}$ or $15 \mathrm{MV}$ plan could be substituted with $15 \mathrm{MV}$ or $10 \mathrm{MV}$ respectively while maintaining the change to the PTV dose of less than $2 \%$. These findings could be used as a general rule of thumb only for treatment plans generated using the 3DCRT technique in conjunction with the collapsed cone convolution algorithm and not for other techniques such as IMRT or VMAT.

These results were then verified using ion chamber measurements and comparing to the TPS results. It was seen from Table 4 that the difference between ion chamber measurements and the treatment planning calculations were $1 \%$ or less, this can be attributed to the relative output for $10 \mathrm{MV}$ and $15 \mathrm{MV}$ being $1 \%$ above the ideal.
Neutron dose from the $15 \mathrm{MV}$ beam was not considered in this study as it has previously been shown that the absolute lifetime risk of malignancies caused by secondary neutrons in a $15 \mathrm{MV}$ beam during intensity modulated radiation therapy (IMRT) only increased slightly compared to the risk for a 6MV IMRT treatment. ${ }^{9}$ 3DCRT has only a portion of the MU's delivered during IMRT and thus considered to have even less risk than IMRT treatment plans. The results here indicate the factors which need to be considered when substituting energy in for the planned energy are the number of fractions which will have a substituted energy as a percentage of the overall treatment.

Whilst beam substitution is not the recommended approach to treat patients when they are unable to be treated with the planned energy, this technical study suggests that patient dosimetry is not greatly affected if a treatment plan of $15 \mathrm{MV}$ or $10 \mathrm{MV}$ were to be substituted with $10 \mathrm{MV}$ or $15 \mathrm{MV}$ respectively for up to a fifth of the number of fractions.

\section{Conflict of interest}

The authors declare that they have no conflicts of interest. The authors alone are responsible for the content and writing of the paper.

\section{References}

1. Varian Medical Systems 2013 Available from: http://www.varian.com/us/oncology/radiation oncology/clinac/.

2. Weiss E, Siebers JV, Keall P J. An analysis of $6 \mathrm{MV}$ versus $18 \mathrm{MV}$ photon energy plans for intensity-modulated radiation therapy (IMRT) of lung cancer. Radiother Oncol 2007; 82: $55-62$.

3. St-Hilaire J, Sevigny C, Beaulieu F, Gingras L, Tremblay D, Beaulieu L. Optimisation of Photon beam energy in aperture based inverse planning. J Appl Clin Med Phys 2009; 10: 3012.

4. Molazeda M, Saberi H, Rahmatnezhad L, Molani A, Jabbari A. Evaluation the effect of photon beam energies on organ at risk doses in three-dimensional conformal radiation therapy. Research Journal of Applied Science, Engineering and Technology 2013; 6:2110-2117.

5. Philips Radiation Oncology Systems. Pinnacle v9.2. Fitchburg, WI: 2013

6. Wellhofer Dosimetrie, inventor; IC-15. Schwrazenbruck Germany. 
7. Pokharel S. Dosimetric impact of mixed- energy volumetric modulated arc therapy plans for high risk prostate cancer. International Journal of Cancer Therapy and Oncology 2013; 1(1):01011.

8. Bestheda MD. Prescribing, recording and reporting photon beams. International Commis- sion of Radiation Units and Measurements (ICRU) Report No. 50, 1993.

9. Kry SF, Salehpour M, Followill DS, et. al. The calculated risk of fatal secondary malignancies from intensity modulated radiation therapy. Int J Radiat Oncol Biol Phys 2005; 62: 1195-203. 